\title{
Blood component therapy, demographic and outcome feature of pediatric acute lymphoblastic leukemia
}

\author{
Mehran Noroozi $^{\circledR}$, Farid Ghazizadeh ${ }^{2 *(\mathbb{D}}$, Saba Fani $^{(\mathbb{D}}$ \\ 'Department of Pediatrics, Faculty of Medicine, Urmia University of Medical Sciences, West Azerbaijan, Iran \\ ${ }^{2}$ Department of Biostatistics, Faculty of Medicine, Urmia University of Medical Sciences, West Azerbaijan, Iran \\ ${ }^{3}$ Faculty of Medicine, Urmia University of Medical Sciences, West Azerbaijan, Iran
}

Correspondence to:

Farid Ghazizadeh; Email:

ghazizadeh.f@umsu.ac.ir

Received: 14 May 2021

Accepted: 10 August 2021

ePublished: 1 September 2021

Keywords: Acute lymphoblastic leukemia, Blood component therapy, Demographic

\begin{abstract}
Introduction: Acute lymphoblastic leukemia (ALL) is the most common childhood malignancy, with an annual incidence rate of three to four cases per 100000 children. Most children with ALL frequently receive blood products including packed cells, platelets, fresh frozen plasma (FFP) and whole blood in the course of chemotherapy and these transfusions may affect ALL outcomes.

Objectives: This study aimed to evaluate blood component therapy together with demographic and outcome features of pediatric ALL patients.

Patients and Methods: Demographic information of 208 patients with pediatric ALL from February 2011 to August 2019 enrolled in this cross-sectional study. Data is gathered and rechecked from archive files and e-files of Motahari hospital.

Results: The mean age of patients at diagnosis was $5.48 \pm 3.38$ years and Pre-B ALL was the most common phenotype (94.3\%). 130 Out of 208 patients were treated with the new protocol and 78 patients were treated with the BFM98 protocol. The majority of relapses were in the bone marrow. The average of received packed cell, platelet and FFP were $4.32 \pm 2.93,5.97 \pm 7.09$ and $5.29 \pm 6.6$ units, respectively. The mean overall survival of patients was $3.42 \pm 2.58$ years in 10 years.

Conclusion: According to this study, most of the patients were 5 to 15 years old. Dominant subtype of disease was B-cell type. Most of the deaths were one to 6 years after diagnosis. The relapse rate was about $31 \%$ and most of them were in the bone marrow.
\end{abstract}

\section{Introduction}

Approximately 6000 cases of acute lymphoblastic leukemia (ALL) are diagnosed in the United States each year. Half of the cases occur in children and adolescents. In the United States, ALL is the most common cancer among children and the leading cause of cancer death before the age of $20(1,2)$.

Symptoms of ALL include bruising or bleeding from thrombocytopenia, paleness and fatigue from anemia, and post-neutropenia infection. Leukemic infiltration of the liver, spleen, lymph nodes and mediastinum may help to make diagnosis. Extramedullary ALL in the central nervous system (CNS) or testes may require special treatment modifications (3). The first description of the temporary cure of leukemia by chemotherapy was in 1948 (4). Clinical characteristics of the patients, biological characteristics of leukemic cells and early response to treatment, prediction of the risk of recurrence and modifying

\begin{abstract}
Key point
Blood products transfusion is the main supportive care in the course of chemotherapy of leukemia patients. Despite their efficacy and patients' need to packed cell, platelet, fresh frozen plasma transfusion, it should be kept in mind that over-transfusion of each of products will result in several side effects that put patients cure at risk. Gathering initial data about the amount of blood products transfusion is the first and most important pillar in management of products transfusion in the future. Our study on 208 patients with pediatric acute lymphoblastic leukemia showed the most common subtype of disease was B-cell. Most of deaths were 1 to 6 years after diagnosis. The relapse rate was about $31 \%$ and the most common site of relapse was bone marrow.
\end{abstract}

chemotherapy intensity provides a model for improving survival in cancer patients. Overall, these advances have improved survival rates from less than $10 \%$ in the 1960 s to $90 \%$ today (3).

Allogeneic blood transfusion exposes the receptor to a wide range of soluble and

Copyright $\odot 2021$ The Author(s); Published by Society of Diabetic Nephropathy Prevention. This is an open-access article distributed under the terms of the Creative Commons Attribution License (http://creativecommons.org/licenses/by/4.0), which permits unrestricted use, distribution, and reproduction in any medium, provided the original work is properly cited. 
mediated alloantigens, cytokines, and other cellular components. Allogeneic blood transfusion is associated with effects on the immune system in the form of complete immunization or suppression of the immune system (5). This complex interaction between the blood component and the host immune system is known as transfusionrelated immunomodulation (TRIM). TRIM was initially found to have a beneficial effect by increasing the survival of renal allografts in patients (6). However, the effect of TRIM was later associated with tumor growth, bacterial degradation, postoperative mortality, and organ dysfunction $(7,8)$.

\section{Objectives}

This study aimed to evaluate blood component therapy together with demographic and outcome features of pediatric ALL patients.

\section{Patients and Methods}

\section{Study design}

This study is a descriptive cross-sectional study performed on pediatric patients with ALL. All patients who were diagnosed with ALL from February 2011 to August 2019 in Shahid Motahari hospital and their documents were recorded in the hospital electronic file were enrolled in the study. Patients who died before a definitive diagnosis or who refused to continue treatment or their documentation was not fully recorded were excluded from the study. Variables such as age, gender, high or low risk, treatment methods, bone marrow, testicular or CNS relapses were evaluated.

\section{Data analysis}

SPSS version 20 was employed to statistically analysis of gathered data. Descriptive values such as percentages, frequencies, mean \pm standard deviation, interquartile range and median, were used. To statistically analysis of data, paired $t$ test and Wilcoxon tests were used, depending on the distribution of quantitative data as determined by the Kolmogorov-Smirnov test. Correlation was assessed using the Pearson's coefficient. $P$ values $<0.05$ were deemed statistically significant.

\section{Results}

The mean age of patients was $9.57 \pm 4.42$ years. The subjects were 105 males (50.2\%) and 103 females (49.3\%). The mean age of patients at diagnosis was $5.45 \pm 3.38$ years (range, 11-14 years). Around 62.2\% $(\mathrm{n}=130)$ of patients were treated with the new protocol and $37.8 \%$ of patients with the BFM 98 protocol.

The cellular subtype of leukemia in most of patients was B-cell type (94.3\%) followed by T-cell (5.2\%) and chronic myeloid leukemia (0.5\%)in 10 patient and 1 patient, respectively.

The mean number of received pack cells was $4.32 \pm 2.93$ units, the mean number of platelets received was 5.97. \pm 7.09 unit, the number of received plasma was $5.29 \pm 6.6$ units. About 131 patients (64.9\%) were in the low-risk group and 77 were in the high-risk group (Table 1).

The mortality rate of patients in low risk and high risk groups was compared by Kolmogorov-Smirnov statistical test and independent $t$ test. The test showed that there was a significant difference in mortality rate of low-risk and high-risk groups. Analyzing of mortality rate based on gender variable and treatment protocol showed that there was no significant difference in mortality rate of male and female patients also between BFM98 and new protocol $(P$ $>0.05)$.

The mean 10- year overall survival (OS) of patients was $6.94 \pm 1.08$ years. The median survival of patients was 7 years. Patients' OS was compared based on the treatment protocol. The test results showed that there was no significant difference between OS in people with new and old protocol $(P>0.05)$. The mean OS was $5.18 \pm 2.32$ years in subjects with BFM 98 protocol and $4.34 \pm 2.01$ years in subjects with new treatment. Patients' OS was compared based on gender variable and in low-risk and high-risk patients. The test results showed that there was no significant difference between OS in males and females also in low-risk and high-risk patients $(P>0.05)$.

Based on the available information, recurrence was observed in $31 \%$ of patients. The most common site of relapse was bone marrow and then the CNS.

\section{Discussion}

In this study, incidence rate of leukemia in both genders was the same; however, in the study by Hashemi et al the male to female ratio was 1.7:1 (9). Age at diagnosis was recognized as a risk factor for recurrence. Relapse rate was significantly higher in the 5 to 10 years old age group than in the age group under five years. In a study that looked at the consequences of leukemia, age was identified as one of the most important predictors of death (10). However, in the study of Arya et al, no significant relationship was reported between age and recurrence of the disease. Regarding the gender of patients, as in the study by Arya

Table 1. Transfused blood components

\begin{tabular}{lccccc}
\hline & N & Minimum & Maximum & Mean & Standard Deviation \\
\hline Platelets & 122 & 1.00 & 53.00 & 5.97 & 7.09 \\
Fresh frozen plasma & 35 & 1.00 & 34.00 & 5.29 & 4.60 \\
Packed red blood cell & 165 & 1 & 12 & 2.93 \\
\hline
\end{tabular}


et al, in our study, also no significant correlation was observed between gender and rate of relapse (11). Relapse is the most common cause of treatment failure in patients with leukemia (12), which leads to a decrease in survival and an increase in mortality in patients. In the present study, the recurrence rate was about $31 \%$ while in a study in Turkey relapse rate was $25 \%(13,14)$.

\section{Conclusion}

According to this study, most of the patients were 5 to 15 years old and mean age at diagnosis was 5.48 years. The most common subtype of disease was B-cell. Most of deaths were 1 to 6 years after diagnosis. The relapse rate was about $31 \%$ and the most common site of relapse was bone marrow.

\section{Limitations of the study}

In this study, incomplete information of hospitalized patient files was one of the limitations in achieving the desired results. Some information about the history and course of the disease was inconsistent, and some of the information in the files was not verified.

\section{Authors' contribution}

MN, FG and SF were the principal investigators of the study. FG, SF participated in the concept and design. FG revisited the manuscript and critically evaluated the intellectual contents. All authors participated in preparing the final draft of the manuscript, revised the manuscript and critically evaluated the intellectual contents. All authors have read and approved the content of the manuscript and confirmed the accuracy or integrity of any part of the work.

Conflicts of interest

The authors declare that there is not any conflict of interest.

\section{Ethical issues}

The research followed the tenets of the Declaration of Helsinki. The institutional ethical committee at Urmia University of Medical Sciences approved all study protocols (IR.UMSU.REC.1399.350). Accordingly, written informed consent was taken from all participants before any intervention. Moreover, ethical issues (including plagiarism, data fabrication, double publication) have been completely observed by the authors.

\section{Funding/Support}

None to be declared.

\section{References}

1. Smith MA, Seibel NL, Altekruse SF, Ries LA, Melbert DL, O'Leary M, et al. Outcomes for children and adolescents with cancer: challenges for the twenty-first century. J Clin Oncol. 2010;28:2625-34. doi: 10.1200/JCO.2009.27.0421.

2. Linabery AM, Ross JA.Trends in childhood cancer incidence in the USA 1992-2004. Cancer. 2008;112:416-32. doi: 10.1002/ cncr.23169.

3. Hunger SP, Mullighan CG. Acute Lymphoblastic Leukemia in Children. N Engl J Med. 2015;373:1541-52. doi: 10.1056/ NEJMra1400972.

4. Linabery $\mathrm{AM}$, Ross JA.trends in childhood cancer incidence in the U. S. Cancer. 2008;112:416-32. doi: 10.1002/cncr.23169.

5. Blumberg N, Triulzi DJ, Heal JM. Transfusion-induced immunomodulation and its clinical consequences. Transfus Med Rev. 1990;4:24-35. doi: 10.1016/s0887-7963(90)702398.

6. Opelz G. Current relevance of the transfusion effect in renal transplantation. Transplant Proc. 1985;17:1015-21.

7. Landers DF, Hill GE, Wong KC, Fox IJ. Blood transfusioninduced immunomodulation. Anesth Analg.1996;82:187-204. doi: 10.1097/00000539-199601000-00035.

8. Blajchman MA. Transfusion immunomodulation or TRIM: what does it mean clinically? Hematology. 2005;10:208-14. doi: 10.1080/10245330512331390447.

9. Hashemizadeh $\mathrm{H}$, Jafarzadeh A, Broumand H. Risk Factors andthe Most Common Initial Symptoms of Acute Lymphoblastic Leukemia in Children. Iran J Nurs. 2011;24:67-77.

10. Hazar V, Karasu GT, Uygun V, Akcan M, Küpesiz A, Yesilipek A. Childhood acute lymphoblastic leukemia in Turkey: factors influencing treatment and outcome: a single center experience. J Pediatr Hematol Oncol. 2010;32:e317-22. doi: 10.1097/MPH.0b013e3181ed163c.

11. Arya LS, Kotikanyadanam SP, Bhargava M, Saxena R, Sazawal $S$, Bakhshi S,et al. Pattern of relapse in childhood ALL: challenges and lessons from a uniform treatment protocol. J Pediatr Hematol Oncol. 2010;32(5):370-5. doi: 10.1097/ MPH.0b013e3181d7ae0d.

12. Gaynon PS. Childhood acute lymphoblastic leukaemia and relapse. Br J Haematol. 2005;131:579-87. doi: 10.1111/j.13652141.2005.05773.x

13. Szczepanek J, Styczyński J, Haus O, Tretyn A, Wysocki M. Relapse of acute lymphoblastic leukemia in children in the context of microarray analyses. Arch Immunol Ther Exp (Warsz). 2011;59:61-8. doi: 10.1007/s00005-010-0110-1.

14. Hazar V, Karasu GT, Uygun V, Akcan M, Küpesiz A, Yesilipek A. Childhood acute lymphoblastic leukemia in Turkey: factors influencing treatment and outcome: a single center experience. JPediatr Hematol Oncol. 2010;32:e317-22. doi: 10.1097/MPH.0b013e3181ed163c. 